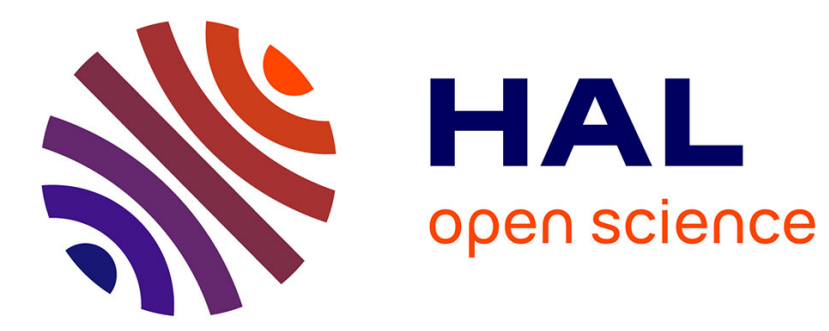

\title{
SPECTRON, a neutron noise measurement system in frequency domain
}

G. de Izarra, C. Jammes, B. Geslot, J. Di Salvo, C. Destouches

\section{To cite this version:}

G. de Izarra, C. Jammes, B. Geslot, J. Di Salvo, C. Destouches. SPECTRON, a neutron noise measurement system in frequency domain. Review of Scientific Instruments, 2015, 86 (11), pp.115111. $10.1063 / 1.4935250$. cea-02382890

\section{HAL Id: cea-02382890 https://hal-cea.archives-ouvertes.fr/cea-02382890}

Submitted on 27 Nov 2019

HAL is a multi-disciplinary open access archive for the deposit and dissemination of scientific research documents, whether they are published or not. The documents may come from teaching and research institutions in France or abroad, or from public or private research centers.
L'archive ouverte pluridisciplinaire HAL, est destinée au dépôt et à la diffusion de documents scientifiques de niveau recherche, publiés ou non, émanant des établissements d'enseignement et de recherche français ou étrangers, des laboratoires publics ou privés. 


\title{
SPECTRON, a neutron noise measurement system in frequency domain
}

\author{
G. de Izarra, ${ }^{1}$ C. Jammes, ${ }^{1,}$ a) B. Geslot, ${ }^{1}$ and C. Destouches ${ }^{1}$ \\ CEA, DEN, Cadarache, Spex/LDCI, F-13108 Saint-Paul-lez-Durance, France
}

(Dated: 4 June 2015)

\begin{abstract}
This paper is dedicated to the presentation and the validation of SPECTRON, a new neutron noise measurement system developed at the CEA centre of Cadarache. The apparatus is designed for the measurement of the $\beta_{\text {eff }}$ parameter (mean fraction of delayed neutrons) from cores of experimental nuclear reactors with the help of Cohn- $\alpha$ method. An integrated electronic system is used to record the current from fission chambers. Data spectra computed from measurement data processed by dedicated software in order to estimate the reactor transfer function and then the mean fraction of delayed neutron as well as prompt neutron generation time. After a review of the pile noise measurement method, the Spectron architecture is presented; Then, the validation procedure is described and experimental results are given to prove the proper operation of our new measurement system. It is shown that every technical requirement needed for proper measurement of neutron noise is fulfilled. Measurements in real condition performed on the experimental CEA's nuclear reactor MINERVE allows validating the good operation of Spectron.
\end{abstract}

\section{INTRODUCTION}

The determination of the $\beta_{\text {eff }}$ parameter with good precision on nuclear reactors' cores is of great importance due to the influence of this parameter on operation safety. Since more than twenty years, a long term work has been carried out at the CEA to enhance the measurement of $\beta_{\text {eff }}$ trough experimental programs and through the conception of measurement devices ${ }^{1,2}$. From this perspective, Spectron, an integrated system for neutronic noise measurement, has been developed at the instrumentation laboratory of the CEA center of Cadarache. The first part of this paper is dedicated to the presentation of the Spectron architecture. At this occasion, the "pile noise" measurement technique is reminded.

The second part of this paper deals with the Spectron validation. Each technical requirement is checked in the laboratory with the help of electrical diagnostic benches and neutron noise simulation apparatus. Then, the system is proven with neutron noise measurement on the MINERVE reactor. The comparison of obtained results with previous experimental and computed ones allows to highlight the advantages of Spectron.

\section{NOTIONS OF NEUTRON NOISE MEASUREMENT}

This section aims at briefly presenting the theoretical background needed to understand the rationale of the so-called neutron noise measurement techniques in a nuclear reactor at zero-power. Zero-power means that the reactor power is about a few tens watts. This way, any fluctuations observed in a neutron detector signal originate from either the counting process ${ }^{3}$ or the fission process ${ }^{4}$. The counting process takes place in detectors whereas the fission process takes place in the whole

\footnotetext{
a) christian.jammes@cea.fr
}

reactor core where the fissile fuel is. Neutron noise measurement techniques deal with those fluctuations.

\section{A. Reactor kinetics}

In a reactor, the neutron flux satisfies the Boltzmann transport equation ${ }^{5}$. This flux is actually a scalar average quantity equal to the neutron density times the neutron velocity. A nuclear reactor in operation exhibits a steady neutron flux without external neutron source: the reactor is said to be at critical. It is important to note that each fission reaction produces prompt and delayed neutrons. The former are promptly emitted whereas the latter come later, after some delay, from fission products that undergo a beta decay immediately followed by a neutron emission. Most of fission neutrons are prompt. Delayed neutrons represents a very small fraction that amounts to a few hundreds of parts per $10^{5}$ or pcm (per cent mille). A fission chain consists of prompt neutrons only. Delayed neutrons play a key role since they initiate new fission chains. One can define the neutron flux as the superposition of individual fission chains. One also shows that the number of prompt neutrons of a fission chain $n_{p}$ satisfies the following time-dependent equation ${ }^{6}$ :

$$
\frac{\mathrm{d} n_{p}}{\mathrm{~d} t}=-\alpha_{p} n_{p}(t)+\frac{1}{\Lambda} s_{d}(t)+\frac{1}{\Lambda} s(t)
$$

with

$$
\begin{aligned}
\alpha_{p} & =\frac{\beta-\rho}{\Lambda} \\
s_{d}(t) & =\sum_{i} \delta\left(t-t_{i}\right) \\
s(t) & =\sum_{j} \delta\left(t-t_{j}\right)
\end{aligned}
$$


and the following kinetics parameters:

- $\rho$ the reactivity that is equal to zero at critical,

- $\beta$ the delayed neutron fraction ;

- $\Lambda$ the neutron time generation, which is the average time between two neutron birth events.

The delayed neutron source $s_{d}(t)$ is intrinsic whereas $s(t)$ is an optional external source. At critical, the number of fission chains is equal to that of delayed neutrons: the fission chain process is self-stationary: there is no use for an external source since delayed neutrons act as an intrinsic source ${ }^{6}$.

The neutron flux is the superposition of fission chains:

$$
\phi=\sum \text { fission chains }
$$

This quantity also satisfies a lumped equation equivalent to Eq. (1). One can note that the neutron flux $\phi$ is a function of time $t$, space $\vec{r}$ and neutron velocity $\vec{v}$. In most of cases, one can factorize the neutron flux as a time-dependent amplitude function $p(t)$ and a timeindependent shape function $\psi(\vec{r}, \vec{v})$ :

$$
\phi(t, \vec{r}, \vec{v})=p(t) \psi(\vec{r}, \vec{v})
$$

This is the so-called point kinetics approximation ${ }^{6}$ that leads to:

$$
\frac{\mathrm{d} p(t)}{\mathrm{d} t}=-\alpha_{p} p(t)+\frac{1}{\Lambda} S_{d}(t)+\frac{1}{\Lambda} S(t)
$$

The delayed neutron source $S_{d}(t)$ is an implicit function of $\mathrm{p}(\mathrm{t}) . \quad S(t)$ is an external source. The neutron flux amplitude is then also governed by the so-called kinetics parameters, namely $\rho, \beta$ and $\Lambda$. A nuclear reactor can be thus viewed as a linear time-invariant system, the time behavior of which is described by either its impulse response in time domain or its transfer function in frequency domain. This functional time model depends on the so-called reactor kinetics parameters.

\section{B. Power spectral density}

The prompt time response of either the neutron number $n_{p}(t)$ of a fission chain or the neutron flux amplitude $p(t)$ is driven by prompt neutrons only. One denotes $\Delta t$ as the timespan between the date $t=0$ where any neutron source is emitted and the date $t+\Delta t$ where the delayed neutrons emission starts. After Eq (1) and (7), it is straightforward that $n_{p}(t)$ and $p(t)$ are linear functions of a time exponential:

$$
n_{p}(t) \text { or } p(t)=a \exp \left(-\alpha_{p} t\right)+b, \quad \text { for } 0 \leq t<\Delta t
$$

where $a$ and $b$ are any real numbers. One recalls that a nuclear reactor is a linear time-invariant system. Its impulse response prior to the delayed neutron emission is thus given by Eq. (8). One notes that this function is decreasing only if $\rho<\beta$ : it corresponds to the operational situation of a nuclear reactor where fission chains are finite.

Since neutron noise measurement techniques use fluctuations, they are actually interested in variations such as

$$
\delta R(t)=R(t)-\langle R(t)\rangle
$$

where $R$ is the count rate obtained by a detector, the neutron interactions of which can be modeled by a macroscopic neutron cross section $\Sigma_{d}$ :

$$
\begin{aligned}
R(t) & \left.=\int \Sigma_{d}(\vec{r}, \vec{v}) \phi(t, \vec{r}), \vec{v}\right) \mathrm{d}^{3} r \mathrm{~d}^{3} v \\
& =p(t) \int\left(\Sigma_{d}(\vec{r}, \vec{v}) \psi(t, \vec{r}, \vec{v}) \mathrm{d}^{3} r \mathrm{~d}^{3} v\right.
\end{aligned}
$$

$\langle$.$\rangle denotes the time average knowing that a nuclear reac-$ tor is assumed to be ergodic. In addition, Eq. 10 shows that $R$ is proportional to the neutron flux in the point kinetics approximation is valid.

Let us apply the time cross-correlation function $C_{i, j}(t)$ to the fluctuations of two detectors $R_{i}$ and $R_{j}$

$$
C_{i, j}(t)=\int \delta R_{i}(u) \delta R_{j}(u+t) \mathrm{d} u
$$

where $t$ is a time lag. One recalls that the counting processes taking place in the two different detectors are uncorrelated. On the contrary, the fission chains, which exhibit the same time behavior expressed by $\exp \left(\alpha_{p} t\right.$ and contribute to the signal, are correlated. Knowing that the neutron flux is a superposition of fission chains, the following one-sided correlation comes

$$
\begin{aligned}
C_{i, j}(t) & \propto \int_{0}^{\infty} \mathrm{e}^{-\alpha_{p} u} \mathrm{e}^{-\alpha_{p}(u+t)} \mathrm{d} t \\
& =\frac{1}{2 \alpha_{p}} \mathrm{e}^{-\alpha_{p} t} \quad \text { for } 0 \leq t
\end{aligned}
$$

The two-sided correlation function is

$$
C_{i, j}(t) \propto \frac{1}{2 \alpha_{p}} \mathrm{e}^{-\alpha_{p}|t|}
$$

It is straightforward to partly obtain the cross power spectral density (CPSD) $S_{i, j}(\omega)$ of $R_{i}(t)$ and $R_{j}(t)$

$$
S_{i, j}(\omega)=\mathcal{F}\left[C_{i, j}(t)\right] \propto \frac{1}{\alpha_{p}^{2}+\omega^{2}}
$$

where $\mathcal{F}$ is the Fourier transform operator, and $\omega$ is the angular frequency. In Ref. ${ }^{4}$, the complete CPSD expression is derived

$$
\begin{aligned}
S_{i, j}(\omega) & =\mathcal{F}\left[C_{i, j}(t)\right] \\
& =\frac{D}{(\beta-\rho)^{2} F_{0}} \times \frac{1}{1+\left(\omega / \alpha_{p}\right)^{2}}
\end{aligned}
$$


with $D$ the Diven's factor and $F_{0}$ the total rate of fissions per second in a nuclear reactor. The Diven's factor is a sort of shape parameter of the distribution of the fission neutron yield $\nu$ :

$$
D=\frac{\mathrm{E}[\nu(\nu-1)]}{\mathrm{E}[\nu]^{2}}
$$

where $\mathrm{E}[$.$] is the expected value.$

\section{Standard instrumentation}

The neutron noise is usually recorded in reactor's core by fission chambers. The standard instrumentation for recording power spectral density is made of a tunable high voltage power supply for fission chamber polarisation, a pre-amplification box in order to convert the low current originating from fission chamber to a measurable voltage and a spectral analyzer to process the measurement and generate PSD or CPSD. Finally, voltmeters are used to have access to the mean voltage value. This instrumentation has many drawbacks; first, its set-up is complex and could lead to errors. Moreover, the use of voltmeter to access the mean value of signal is non convenient and may prevent the detection of low frequency power drift. Secondly, this instrumentation gives only access to mean CPSD or PSD over the measurement time, which make the data analysis difficult, especially is something went wrong during the measurement.

\section{SPECTRON OVERVIEW}

\section{A. Logical and physical architectures}

Spectron is an integrated system developed by the instrumentation laboratory of the CEA's Nuclear studies department in order to replace an obsolete set of devices ${ }^{7}$. It is constituted of an electronic system dedicated to the measurement of neutron noise in current mode for two different calibres: $\mu A$ and $m A$. A personal computer is used for measurement control and in-line/off-line data processing. The logical architecture of this system is available on the figure ??.

This configuration allows to take advantage of the increase of computer memory and computing power: Raw data can be recorded for hours at high frequency sampling and then processed. Moreover, compared to the complicated and cumbersome previous acquisition chain constituted of voltmeters, pre-amplifier boxes and spectrum analyser, Spectron prevents setup and calibrations errors.

The electronic part of Spectron can be reduced to three main boards as presented in figure 2 . The most critical one, the PN000229 card, is responsible for the polarisation of fission chamber and for the measurement of current intensity coming out of those sensors.

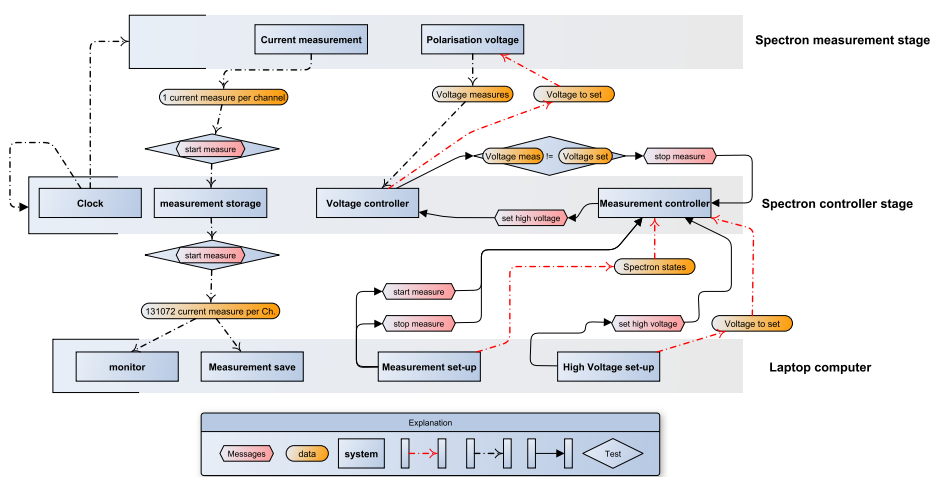

FIG. 1. Logical architecture of the Spectron measurement system.

\section{SPECTRON measurement system}

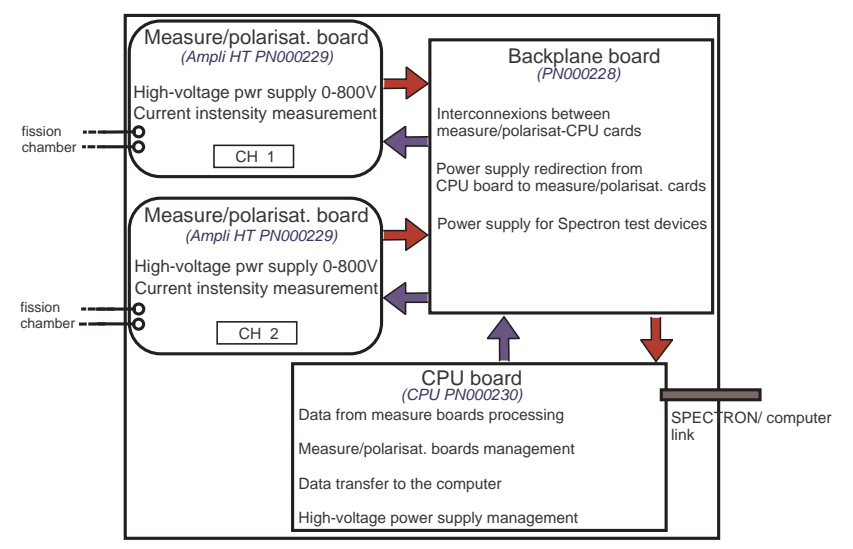

FIG. 2. Schematic of the Spectron Measurement system.

The CPU board is used to control the polarisation voltage and the current measurement. It receives and process data from measure/polarisations boards and finally send them back to the computer.

Besides interconnections for feeding the measurement boards thanks to the CPU board's power supplies, the backplane also contains interconnections for data transmission.

The main components of the measure/polarisation board are shown on the figure 3. Measurements are performed thanks to an $\mathrm{AD} 7763$ analog to digital converter working at a sampling rate of 78125 measures per second. The voltage reference utilized in conjunction to the AD 7763 is a low noise $4.096 \mathrm{~V} \mathrm{ADR} 434 A R Z$. To limit the measurement drift, the AD 7663 is cooled by a Peltier element.

The fission chamber polarisation stage is assured by a DC/DC high voltage converter capable of delivering 3 $\mathrm{mA}$ under $1000 \mathrm{~V}$. This power supply is programmable thanks to a $0-5$ volt input. Polarisation adjustment is performed with the CPU board and is then converted to an input voltage with a $A D 5641 A K S Z$ digital to analog converter. A low resolution ADC is connected at the power supply's probe voltage pin in order to send 
Measure/polarisation board

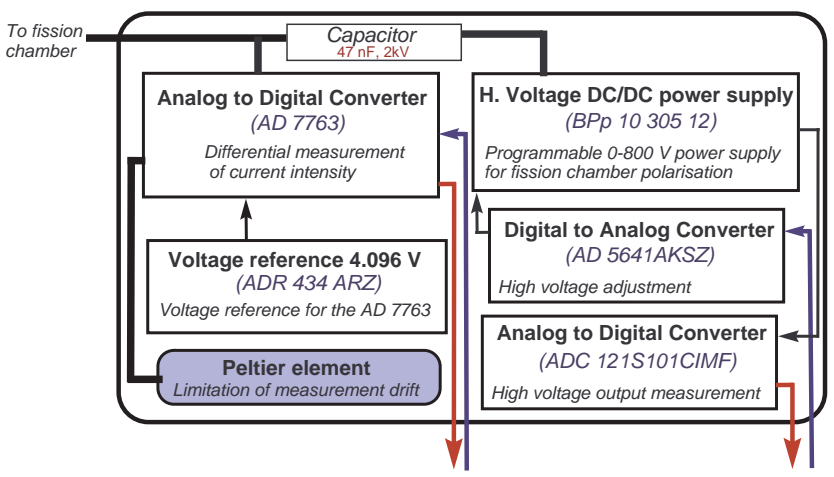

FIG. 3. Schematic of the PN000229 measure/polarisation board. Main components are the $A D 7763$ ADC dedicated for current intensity measurement and the $B P p \quad 10 \quad 30512$ power supply used for the polarisation of fission chamber.

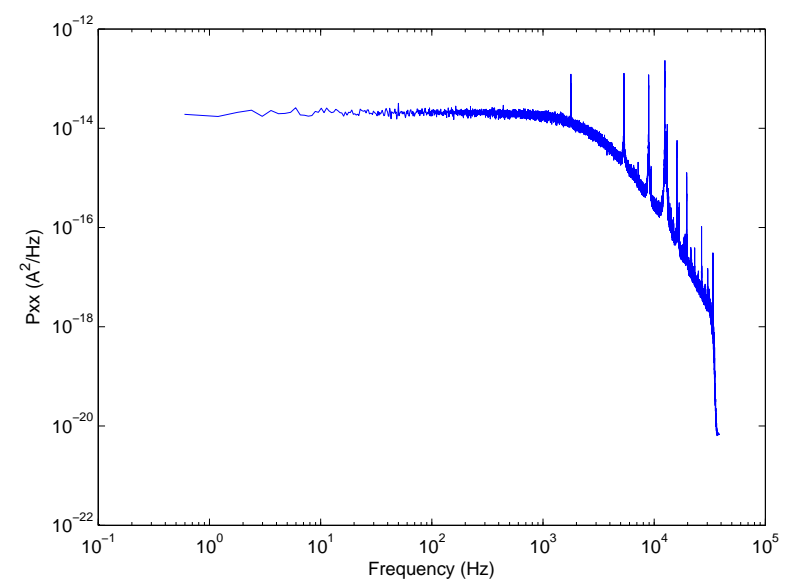

FIG. 4. Power density spectrum of the simulated noise produced with the $1 \mathrm{~mA}$ calibre test tool.

information on fission chamber's polarisation to the CPU card.

In addition to the acquisition chain, two test tools have been constructed in order to quickly check the proper working of the system at the beginning of each measurement campaign. They produce simulated neutron noises for $1 \mathrm{~mA}$ and $1 \mu \mathrm{A}$ measurement calibre with white noise produced by operational amplifiers (MAX4238) and resistors and then filtered by a first order low pass electronic filter. Test tools have a transfer function similar to the one given in relation ?? (fig.4).

\section{B. Data acquisition}

The electronic system is controlled by a simple graphical software. It allows first to setup the measurement parameters like the number of used channels, the fis- sion chambers' polarity and the current intensity measurement calibres. The program also handles the data transfer from the electronic rack. Data are transferred by packets of 131072 measures, which corresponds to roughly 1.67 second of measurements at a sampling rate of $78125 \mathrm{~Hz}$. To assess the quality of the ongoing measure, the software is capable to display raw data as well as mean current intensity and power spectrum density in real time.

The duration of a measurement should be a few hours to ensure a good spectrum's variance. The control software writes measures on the hard drive as a multitude of binary files of about $10 \mathrm{MB}$ in order to handle such a huge quantity of data. This format allows fast data loading and reduced disk usage (17.9 MB per minute per channel).

\section{Data processing and analysis}

The off-line data processing is provided by a Matlab toolbox developed for the occasion. It includes low and high level routines needed for binary files manipulation, and computation of the PSD, the CPSD and the coherence function. Every spectral density is computed with the help of the Bartlett's method (averaged periodogram $)^{8}$ : a spectrum is computed for every data packet and is used to get the mean spectrum in order to lower the variance.

For the power spectral density computed on $L$ datasets of $N$ points, the variance is given by ${ }^{9}$ :

$$
\operatorname{var}\left(G_{x x}(f)\right) \approx \frac{G_{x x}^{2}(f)}{L}\left[1+\left(\frac{\sin (N \pi f)}{N \sin (\pi f)}\right)^{2}\right]
$$

To have a relative variance of $1 \%, 10000$ datasets are required. It corresponds to a measurement of almost five hours.

If a cross power spectral density is considered, the variance is given $b^{9}$ :

$$
\operatorname{Var}\left[\left|G_{x y}(f)\right|\right]=\frac{G_{x y} \cdot G_{x y} *}{\gamma_{x y}^{2}},
$$

where $G_{x y}$ is the CPSD, and $\gamma_{x y}^{2}$ the coherence function defined by:

$$
\gamma_{x y}^{2}=\frac{\left|G_{x y}\right|^{2}}{G_{x x} G_{y y}}
$$

\section{VALIDATION IN LABORATORY ENVIRONMENT}

Spectron had to be validated before being used on nuclear reactor. In this regard, critical technical requirements were checked in the laboratory, and the global op- 


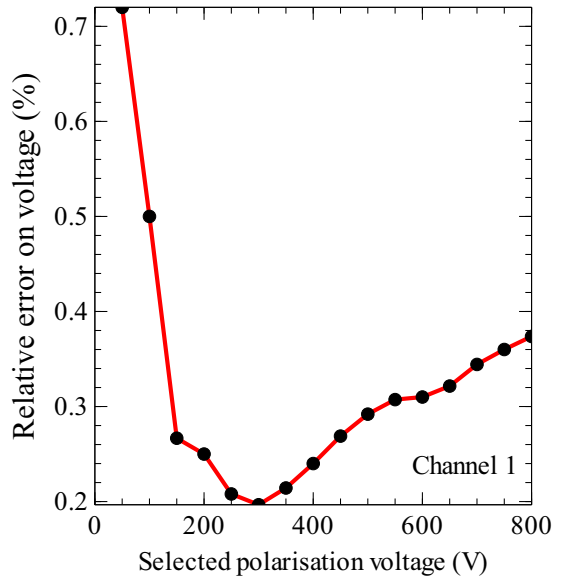

FIG. 5. Relative polarisation voltage error $\left(\frac{\left|V_{\text {meas }}-V_{\text {set }}\right|}{V_{\text {set }}}\right)$ measured for the channel 1. Obtained results for channel 2 are similar.

eration of the system were proved with the help of a neutron noise simulator bench.

The technical requirements Spectron must fill to correctly measure the neutron noise are the following:

- Steady and adjustable polarisation voltage.

- Accurate current measurement for $1 \mathrm{~mA}$ and $1 \mu \mathrm{A}$ calibres with a constant electronic transfer function over the spectral domain of interest.

- Continuous and synchronised measurement; accurate sampling frequency; lossless data transfer.

- Proper evaluation of auto and cross power spectral density.

Each technical requirement involves numerous components from control and post processing softwares to CPU Board and power supplies. Except in special cases, performed tests don't allow to validate the good operation of a single component.

\section{A. Bias voltage application}

The accuracy check of polarisation voltage adjustment were performed with a simple multimeter connected at the input of each measurement board. We made the polarisation voltage varies between 0 and $800 \mathrm{~V}$ with the control software and measured it thanks to the multimeter. The adjustment error found is within the $1 \%$ confidence margin given by the power supply constructor (fig.5). The steadiness of the power supply was check with an oscilloscope and a voltage divider; whatever the selected voltage, the maximum amplitude of fluctuations is $5 \mathrm{mV}_{p p}$ which is much lower than the $40 \mathrm{mV}_{p p}$ maximum fluctuation given by the power supply datasheet.

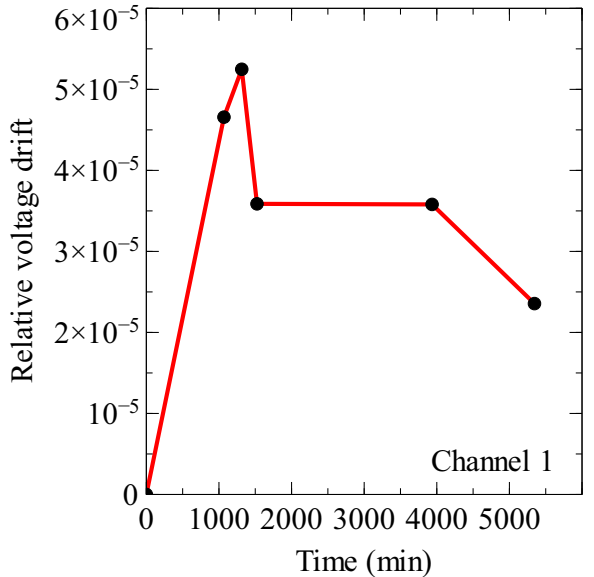

FIG. 6. Relative drift of polarisation voltage over time. The whole experiment was conducted with a $800 \mathrm{~V}$ polarisation voltage.

To ensure the stability of the polarisation voltage over the time of a measurement which is roughly five hours, it was decided to the set operating voltage to $800 \mathrm{~V}$ and takes measurement during a 3.5 days period. It appears that voltage drift is negligible with a maximum relative drift of about $0.006 \%$ (see fig.6).

It has been shown that polarisation voltage characteristics allows working with fission chamber in current mode without any problem since fluctuation are orders of magnitude too low to change the working mode of fission chamber.

\section{B. Data acquisition}

Several technical exigence are required to acquire noise measurement properly; The current measurement should be done accurately with a constant electronic transfer function over the $0-20 \mathrm{kHz}$ spectral domain. Moreover, the measurement should be done in continuous way with a regular sampling. Both requirement involves the ADC/ ADC filter block, the clock, and the CPU card. The validation of each component is impossible, so we decided to check first the accuracy of analog to digital conversion through study of quasi-steady signal. Then the electronic transfer function was probed with wide frequency band signal. At last, the measurement continuity and the sampling accuracy were checked.

\section{Analog-to-digital conversion}

The accuracy of current measurement have been validated by two means. First, a Keithley k6221 current generator have been used to generate stair sweeps. This generator is capable of delivering current in a $4 \mathrm{p} A_{p p}-210$ $\mathrm{mA} p p$ range and is able to generate arbitrary signals; It 

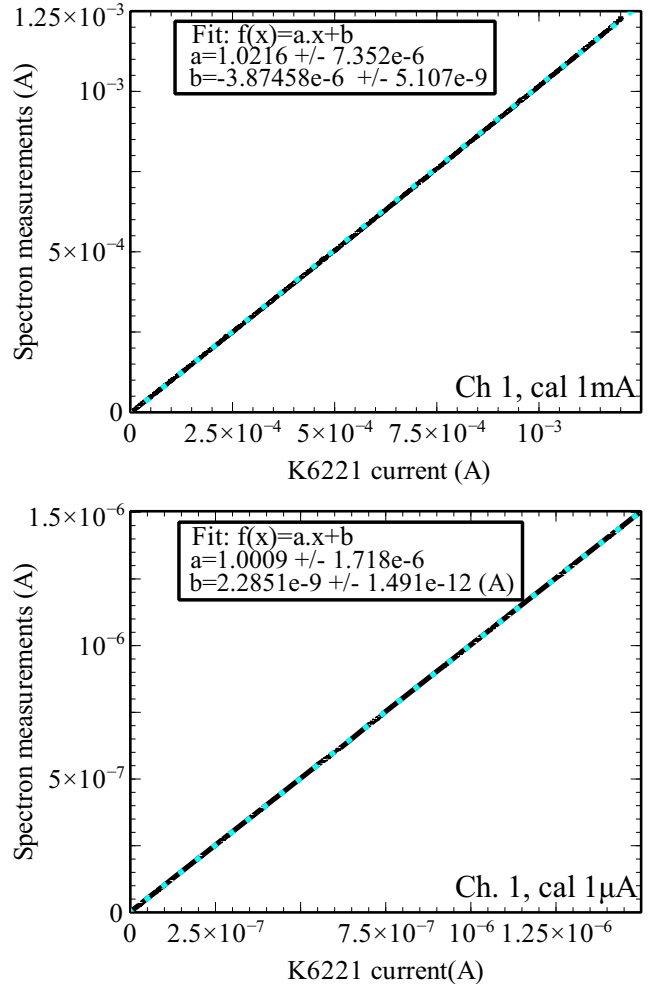

FIG. 7. Linearity and offset of mean current measurement for the channel 1 . The small non linearity of current measurement for the milli-Amp calibre is due to a crude calibration.

is then appropriate for probing the micro-Amp and milliAmp calibres. The measurement linearity and offset were evaluated by plotting $i_{m e s}(t)$, the measured current intensity, versus $i_{\text {deliv }}(t)$ the delivered current and by performing a linear fit. Fit's results are available on the figure 7 ; the measurement offset is negligible whatever the considered calibre. The linearity error is below $1 \%$ for the micro-Amp calibre while it is about $2.2 \%$ and $1.5 \%$ respectively for the milli-Amp calibre of channel 1 and 2. A better calibration should be considered for the milli-Amp calibre to reduce the linearity error and the offset.

In a second time, the electronic transfer function was probed. Since we are interested in the spectrum of measured data, it has to be constant in the frequency domain of interest which is typically $0.1 \mathrm{~Hz}-10 \mathrm{kHz}$. Several methods are available for the measurement of transfer function. Delta Dirac or well known, wide frequency range signal should be used as an input to get the response spectrum of the electronic system. For the sake of simplicity, linear chirps with starting frequency of 1 $\mathrm{Hz}$ and a final frequency of $10 \mathrm{kHz}$ were used. Energy Density Spectrum of linear chirp, if Gibbs phenomena is neglected, is almost flat between the starting and the final frequency. It should be approximated by ${ }^{10,11}$ :

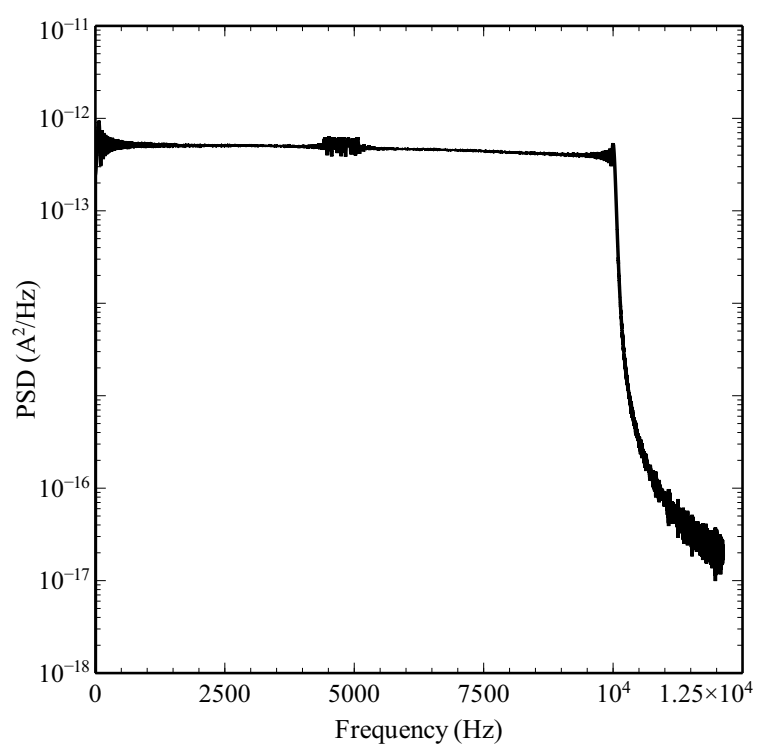

FIG. 8. PSD recorded by Spectron with a $1.67 \mathrm{~s}$ linear chirp from $1 \mathrm{~Hz}$ to $10 \mathrm{kHz}$ as an input on the channel one with the milli-amp calibre. The Keithley k6221 generator was used to produce the signal.

$$
<C_{c}^{2}>=\frac{A^{2} T}{\left[4\left(f_{1}-f_{0}\right)\right]} \text { with } f_{0}<f<f_{1} .
$$

$A$ is the amplitude of the chirp and $T$ is its duration.

Once again, the Keithley k6221 current generator was used to generate linear chirps with a 1.6777 second length for both milli-amp and micro-amp calibres. Results are available on the figure 8 , it shows an almost flat power spectral density between $1 \mathrm{~Hz}-10 \mathrm{kHz}$. The mean spectrum level between $1 \mathrm{kHz}$ and $2 \mathrm{kHz}$ was evaluated to get rid of the influence of Gibbs phenomena and to have the approximation (23) verified; it gives:

$$
\begin{aligned}
& <C_{c}^{2}>_{\text {mes }}=5.1010^{-13} A^{2} / H z . \\
& <C_{c}^{2}>_{\text {theo }}=5.0010^{-13} A^{2} / H z .
\end{aligned}
$$

The same procedure was performed with a waveform generator Stanford Research DS 345 with a built-in chirp function. This time, linear chirp with a 16.777 second duration were recorded with Spectron. Some discrepancies due to the generator are visible for the $1 \mathrm{~Hz}-20 \mathrm{~Hz}$ range, however, the mean spectrum level between $1 \mathrm{kHz}$ and 2 $\mathrm{kHz}$ measured is (fig. 9):

$$
\begin{aligned}
& <C_{c}^{2}>_{\text {mes }}=1.91 \quad 10^{-11} A^{2} / \mathrm{Hz} . \\
& <C_{c}^{2}>_{\text {theo }}=1.90 \quad 10^{-11} A^{2} / \mathrm{Hz} .
\end{aligned}
$$

Results obtained with the DS 345 are better because the duration of chirp makes the approximation 23 more valid. In conclusion, the Spectron's function transfer is flat over the spectral domain of interest, neutron noise should be recorded without any correction. 


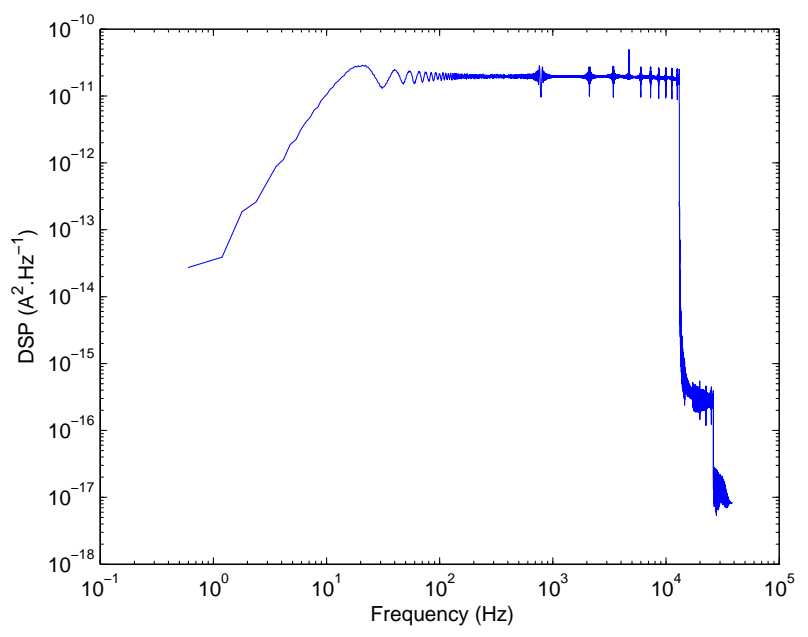

FIG. 9. Power spectrum density recorded by Spectron with a $16.7 \mathrm{~s}$ linear chirp from $1 \mathrm{~Hz}$ to $10 \mathrm{kHz}$ as an input on the channel one with the milli-amp calibre. The Stanford research DS 345 generator was used to produce the signal.

\section{Signal sampling}

The electronic rack records the current properly and provides a steady polarisation voltage. However, the proper data transfer and measurement continuity has to be validated, as well as the accuracy of data sampling.

The validation of those technical requirements were done with the help of a waveform generator; The DS 345 was set in order to generate a $20 \mathrm{kHz}$ sine as an input for Spectron. The signal frequency is far below the Nyquist's one, but is high enough to allow the study of measurement continuity, lossless data transfer and proper sampling.

A three minute long records of the sine signal were taken with Spectron. In order to check the continuity of the measurement, data packets boundaries were examined. No signal jump or distortion is distinguishable whatever the studied boundary.

\section{Data transfer}

Concerning the lossless data transfer, a major part of the 108 recorded data packets have been studied and no proof of missing data points were found.

To validate the sampling frequency accuracy for each channel, the same $20 \mathrm{kHz}$ sine signal was used. The clock uncertainty of the signal generator $(5 \mathrm{ppm})$ is negligible compared to the one of the SPXO018039 oscillator of Spectron. A Matlab script was used to compute the total number of sine period on the measurement. It gives access to the measurement time which is:

$$
T_{P e r}=181.1957 \pm 0.0009 \mathrm{~s}
$$

The measurement duration estimated thanks to the Spectron oscillator is :

$$
T_{\text {spec }}=181.1939 \mathrm{~s}
$$

The relative error on time measurement, $\mathrm{E} t_{r}=9.5$ $10^{-6}$, is in agreement with the data provided by the resonator's manufacturer (maximum relative error of $510^{-5}$ ); the sampling is performed in a proper way.

\section{Data processing}

The procedure used to validate our PSD/CPSD algorithm is well known. First, the spectrum frequency have to be checked. To do that, a sine signal with a well defined frequency $f_{s}$ was computed. Its PSD contain a peak centred around the $f_{s}$ frequency: the spectrum seems to be properly computed. Perseval relationship is used to validate the amplitude of PSD; It states that the energy of a signal is independent of its representation. A one second signal containing a $f_{s}=50 \mathrm{~Hz}$ sine was simulated and it's PSD was computed. The energy computed on the spectrum is equal to 0.5 which prove the correct normalisation of spectrum.

\section{Representative operational conditions}

\section{Low-filtered noise simulation}

To investigate the Spectron behaviour with high immunity cables and the quality of the procedure to extract data from neutron noise, an experimental apparatus dedicated to the neutron noise simulation was constructed. It is made of a keithley k6221 current generator connected to a computer in order to generate arbitrary signal. A Matlab script was written to simulate a Gaussian white noise and then to filter it with a low pass first order filter. Due to the reduced memory of the k6221 generator (65325 points), this script is only able simulate and to load 5.1 seconds of signal (3 complete data packet) at a sampling frequency of $10 \mathrm{kHz}$. To get a good signal variance, the neutron noise simulation routine was placed in a "for" loop, and executed until the desired quantity of data were produced, loaded and played by the k6221 and then recorded by Spectron. To investigate the effect of cable on the measurement quality, the neutron noise simulation apparatus is provided with a set of two high immunity, 11 and 22 meter long FILECA 1209-38 cables, and a set BNC cables.

\section{Operational validation}

The typical cut-off frequency of neutron noise spectrum is around $10 \mathrm{~Hz}-100 \mathrm{~Hz}$ for thermal neutrons reactors, that is why it was chosen to simulate neutron noises 


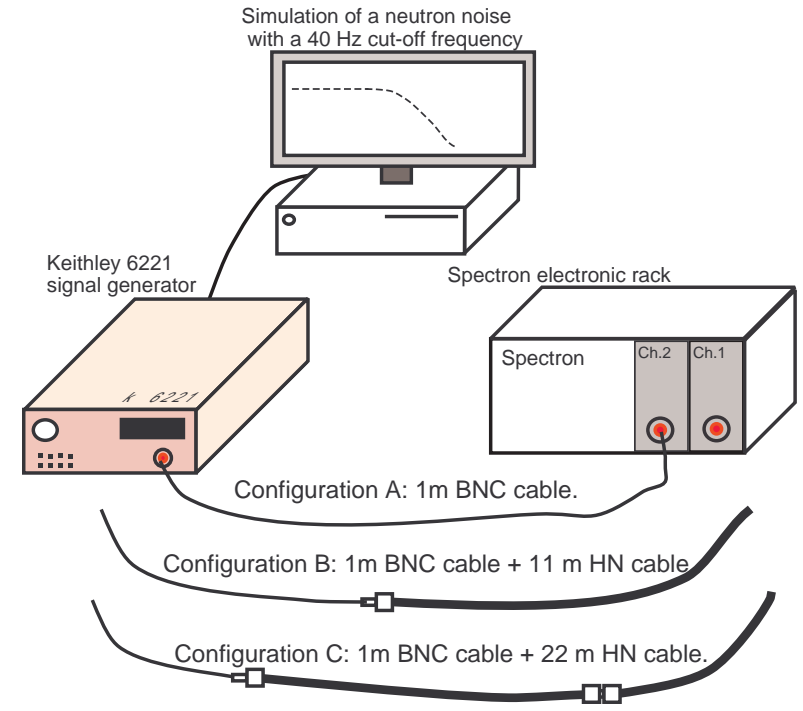

FIG. 10. Experimental set-up developed for neutron noise simulation. The neutron noise is simulated with the help of a MatLab script: a Gaussian white noise filtered with a first order low pass filter. The signal is send to the k6221 generator and then played through different cables connected to Spectron

with a cut-off frequency of $40 \mathrm{~Hz}$. Amplitude of the noises were $11.2 \mathrm{nArms}$ and $11.2 \mu \mathrm{Arms}$ in order to probe the two measurement calibres of Spectron. The effect of high immunity cables used in reactor on signal transmission was also probed during those experiments. The configuration, as well as the experimental set-up used for neutron noise simulation is available on the figure 10 .

Power Spectral Densities, averaged from 80 data packets, are available in fig 10. As it can be seen, the cable length used for signal transmission have no effect at all on the simulated neutron noise's PSD. All the fit performed on noise's spectra give a cut-off frequency of $40 \mathrm{~Hz}$ (for example, the $22 \mathrm{~m}$ long HN cable, micro-amp calibre fit lead to a cut-off frequency of $40 \pm 1 \mathrm{~Hz}$ ).

The complete laboratory validation procedure was successful, the Spectron system can be used in nuclear reactors to retrieve the $\beta_{\text {eff }}$ parameter.

Compared to the old CEA's measurement system, this new tool has several benefits. it's simplicity, it's tuning, and the control software allows to limit the configuration and set-up errors; A non-expert user is now able to carry out a measurement with minimal training. Previously, only the mean cross PSD or PSD were recorded with a low resolution; the memory of the spectral analyser only allows to handle a 1600 points spectrum over a narrow frequency band. With Spectron, the complete raw current intensity measurement are recorded with a sampling frequency of $78125 \mathrm{~Hz}$. Cross PSD and PSD can be computed off-line and on-line and the raw data allows to monitor the measurement off-line in details: if some detectors didn't work properly or if something went wrong during the experimental campaign, useful data still may be ex-
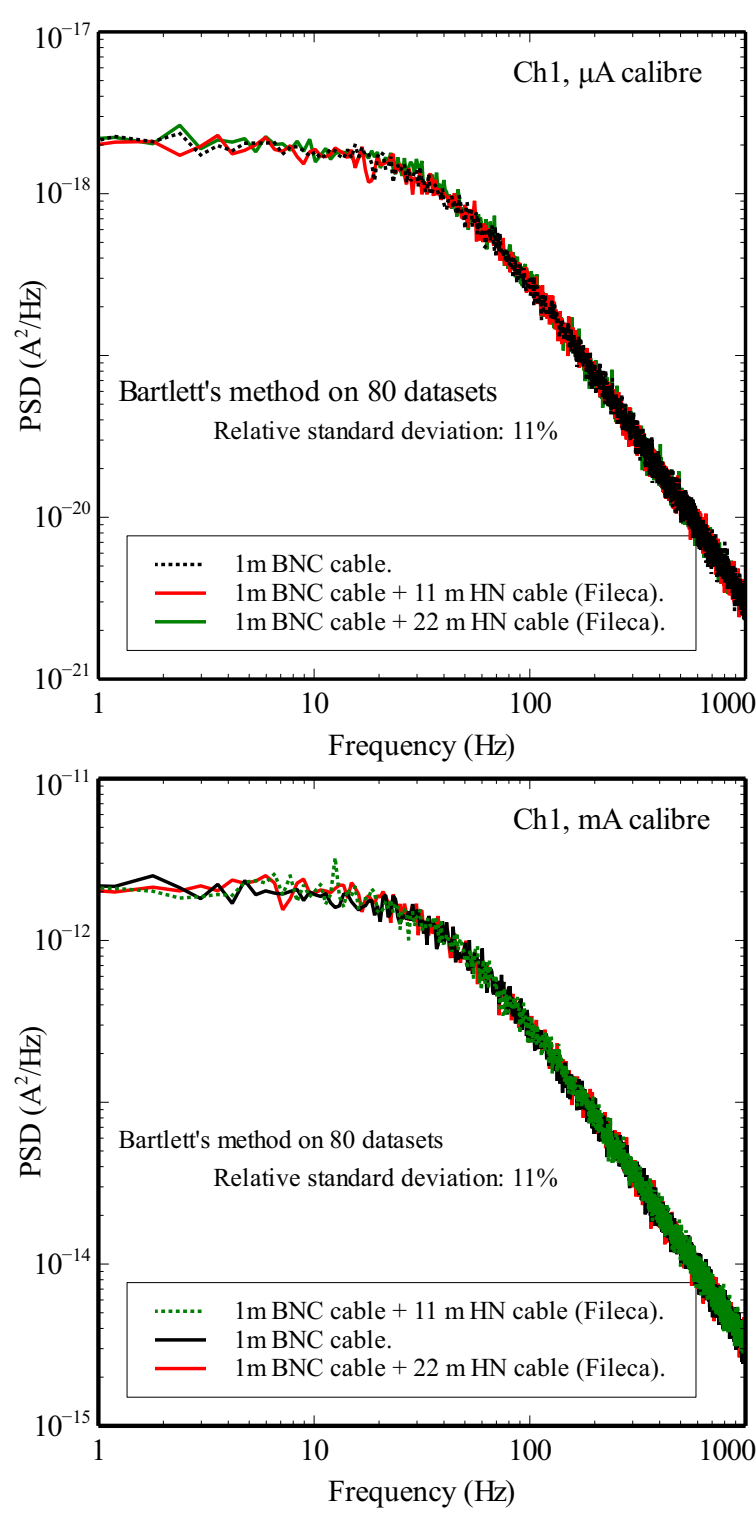

FIG. 11. PSD of the simulated neutron noises with a cut-off frequency of $40 \mathrm{~Hz}$. Three configurations, with different high immunity cables were studied to determine their influence on the transported signal. No difference is noticeable whatever the channel or the calibre used.

tracted from the records. This is of prime interest when we know the difficulty and the cost of planning an experimental campaign. At last, the access to raw data from both channels allows the computation of the coherence function, a measurement of correlated signal contained in the data. It is shown that this quantity is of first importance since it allows selecting the proper frequency domain for kinetic parameter evaluation. Moreover, its computation on preliminary measures make the proper selection of measurement time possible. 


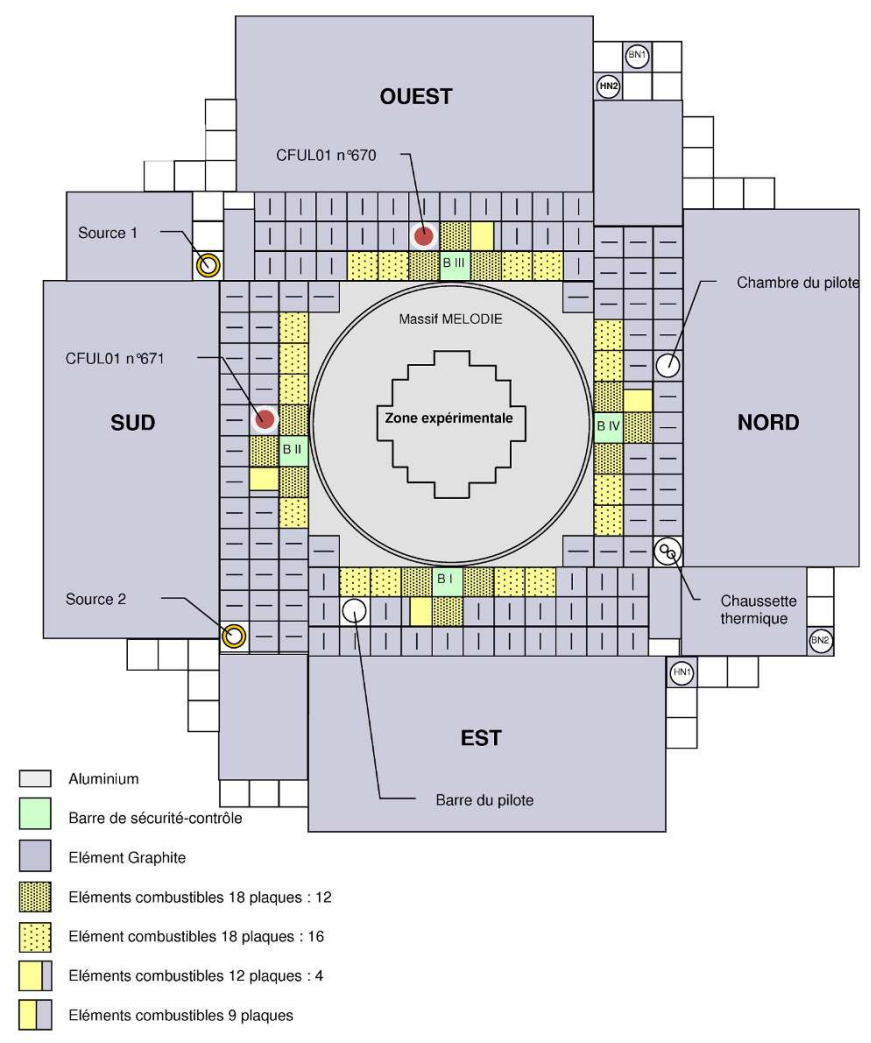

FIG. 12. Schema of the Minerve reactor configuration used during the measurement of kinetics parameters. The CFUL01 number 670 located at the west of the reactor was connected to the Ch.2 of Spectron while the fission chamber number 671 was connected to the Ch.1.

\section{VALIDATION IN REACTOR ENVIRONMENT}

\section{A. MINERVE facility and instrumentation setup}

The Minerve reactor is a french pool type reactor located in the CEA center of Cadarache. It is made of a $120 \mathrm{~m}^{3}$ rhomb-shaped stainless steel tank which contains the reactor core. Its maximum power is about 100 $\mathrm{W}^{12}$. The core, which is submerged under 3 meter of water, is constituted of two different parts; a driver zone that contains aluminium/uranium plate assemblies surrounded by a graphite reflector, and a $70 \mathrm{~cm}$ square hole at the center of the driver zone that receives experimental lattice used for reproducing characteristic neutron spectra. The reactor is operated using four hafnium control rods.

\section{B. Experimental results}

In order to test Spectron and to enhance the incomplete documentation of Minerve's kinetic parameters, an experimental campaign was performed with the MELODIE inner assembly which is dedicated for sim-

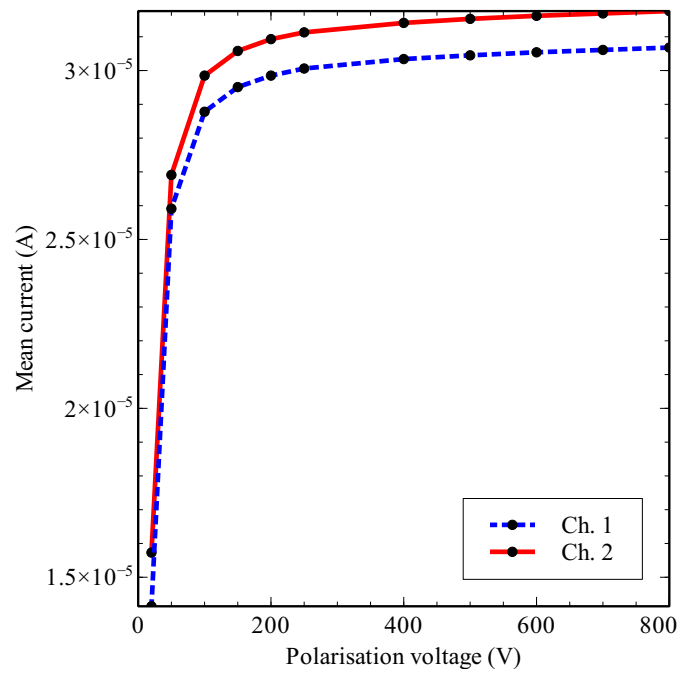

FIG. 13. Calibration curves recorded with the two CFUL01 for a reactor's power of $50 \mathrm{~W}$. Up to $200 \mathrm{~V}$, the recorded current increase sharply with voltage: it's the recombination regime. After $200 \mathrm{~V}$, a plateau is discernible; it corresponds to the saturation regime which is used during neutron noise measurements.

ulating light water lattice. A schema of the reactor's configuration used during the experimental campaign is available in figure 12 . Two CFUL01 with a sensitive length of $211 \mathrm{~mm}$ and $1 \mathrm{~g}$ of $U_{235}$ deposited as a sensitive layer were used to record the neutron noise. The fission chamber labelled 671 was connected to the channel 1 of Spectron, while the 670 fission chamber located in the south part of the reactor was connected to the channel 2.

Calibration curve have been recorded with a reactor power of $50 \mathrm{~W}$ : for different polarisation voltage, the mean current were recorded. Curves are available in figure 13, fission chambers have the same response; below a hundred volts, a sharp increase of current with voltage due to the recombination regime is noticeable. Above $200 \mathrm{~V}$, curves show a plateau which is a sign of the saturation regime. The polarisation voltage was set to $600 \mathrm{~V}$ to use this regime during the whole measurement campaign. The Avalanche regime is not shown because of the Spectron voltage limitation.

To gain confidence about the linearity of the Spectron system, we have recorded neutron noise at different power level. The mean current and the integral of the power spectra density were computed and plotted against the reactor power (fig. 14). It is shown that Spectron is linear for both mean and PSD measurements.

Three long measurements were performed to derive the Minerve's kinetics parameters. Two were critical with a reactor power level of $0.2 \mathrm{~W}$ and $1 \mathrm{~W}$, and one were subcritical. In addition, a long record was conducted with the four control rods down to get the background noise; it allows correcting data from low power measurement.

The $0.2 \mathrm{~W}$ measurement duration was 120 minutes in 

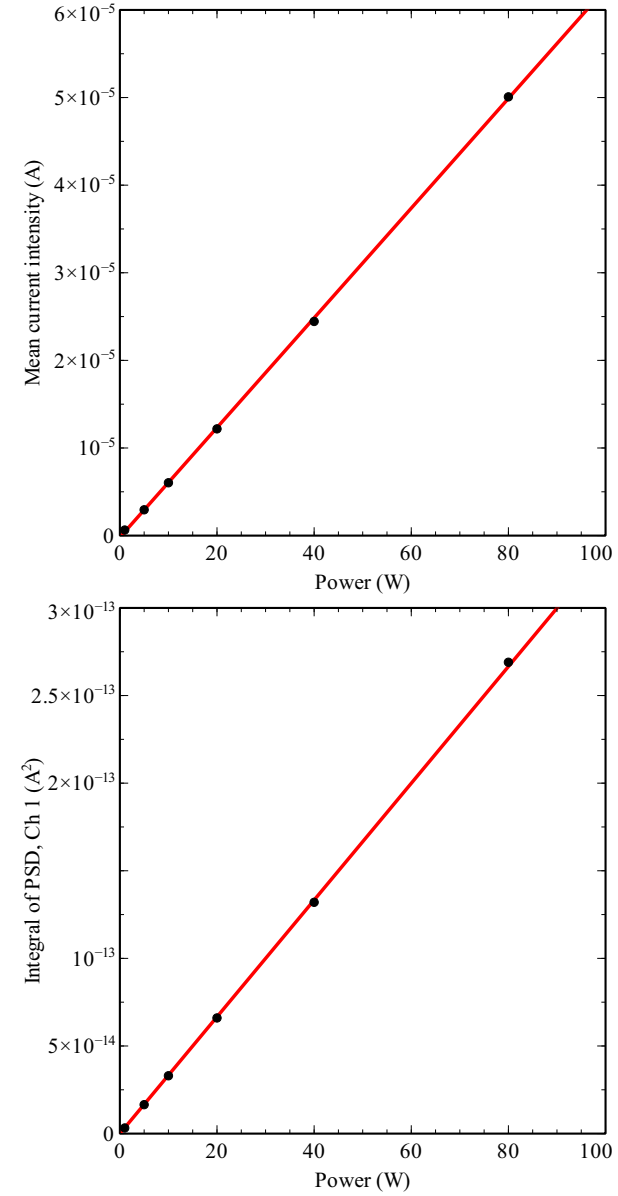

FIG. 14. Upper curve: Mean current (CFUL01 n ${ }^{\circ}$ 671) as a function of the reactor power. Lower curve: Integral of the power spectral density as a function of the reactor power.

order to lower the cross power spectral density variance. The mean current is available on the fig. 15 to assess the stability of the reactor throughout the experiment.

The computed CPSD is shown on the figure 16. The typical reactor transfer function shape is noticeable at for frequency from 0 to a hundred Hertz. The fit of the model was performed on the frequency domain where the computed coherence function is higher than $1 \%$ of its maximum value.

The sub critical measurement was performed to assess the detection limit of Spectron. It's duration was 90 minutes and the mean current recorded on Ch. 1 and 2 were respectively $2.9110^{-8} \mathrm{~A}$ and $2.6510^{-8}$. As the current is close to the offset, a background noise measurement were performed in order to correct the mean current and, if necessary the CPSD. The means current recorded with the four rod down are $1.2110^{-8} \mathrm{~A}$ and 8.36 $10^{-9}$ A respectively for Channel 1 and 2 . The sub-critical reactivity was evaluated with a rod drop experiment, it is $\rho=(0.156 \pm 0.008) \$$.

Kinetic parameters computed are presented on the table I; The given uncertainty were deduced by considering

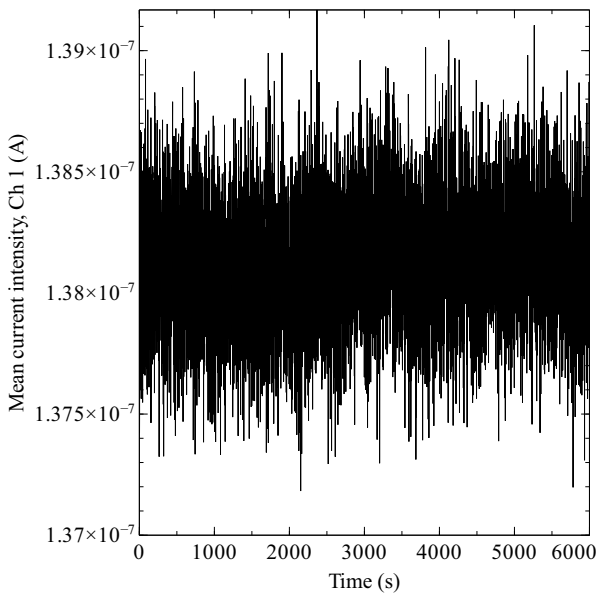

FIG. 15. Mean current recorded on the Ch.1 of Spectron as a function of time. The binning time used to compute the mean was one second.

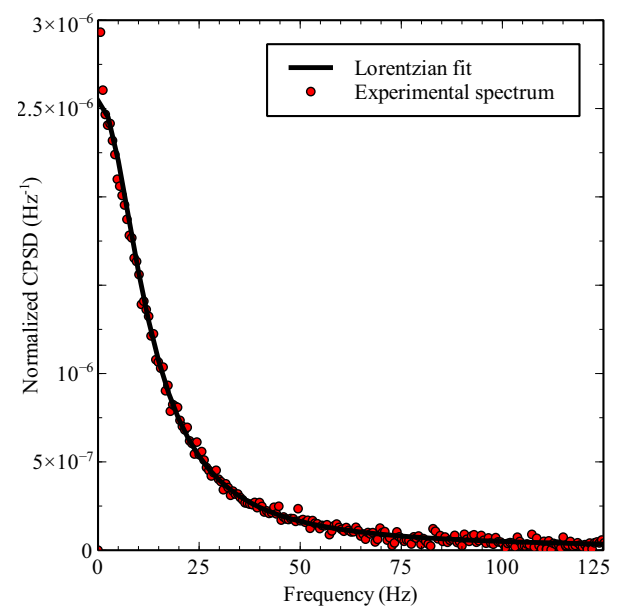

FIG. 16. Normalized CPSD computed with the Bartlett method on the $0.2 \mathrm{~W}$ record. 4293 spectra were averaged to obtain this result.

a $5 \%$ uncertainty on the Diven's factor, on the reactivity and on the fission integral; the uncertainty was computed with the differential method which take into account bias and statistical deviation.. For $0.2 \mathrm{~W}$ and $1 \mathrm{~W}$ measurement, the reactivity was considered equal to zero.

One can see that results obtained with sub-critical data are far from result obtained at $0.2 \mathrm{~W}$ and $1 \mathrm{~W}$ power and possess a larger uncertainty. This can be explained by the weak current level of records which is close to the

TABLE I. Kinectic parameter obtained with the critical and sub-critical measurements.

\begin{tabular}{|l|c|c|c|c|}
\hline Reactor Power & $\mathrm{F} 0(\mathrm{f} / \mathrm{s})$ & $\rho(\$)$ & $\beta_{\text {eff }}(\mathrm{pcm})$ & $\Lambda(\mu \mathrm{s})$ \\
\hline $0.2 \mathrm{~W}$ & $6.7910^{9}$ & - & $715 \pm 40$ & $86 \pm 6$ \\
$1 \mathrm{~W}$ & $3.1010^{10}$ & - & $701 \pm 43$ & $89 \pm 8$ \\
sub-critical & $8.3210^{8}$ & -0.156 & $773 \pm 83$ & $80 \pm 10$ \\
\hline
\end{tabular}


detection limit of Spectron and by the uncertainty which affect the evaluation of reactivity $\rho$. Even in unfavourable measurement conditions, results obtained are close to the expected ones.

Other experimental results obtained on this configuration by G. Perret of the Paul Sherrer Institute with Feynman- $\alpha$ techniques gives the following kinetics parameters $\beta_{\text {eff }}=732 \mathrm{pcm}, \Lambda=89 \mu \mathrm{s}$. Those results are in agreement with the one obtained by Spectron. This show that our new measurement system is reliable and accurate in operating conditions.

\section{CONCLUSION}

The Spectron measurement system, constituted of an electronic rack and a personal computer has been presented. It was constructed in order to replace the obsolete CEA's experimental set-up made of a pre-amplifier box connected to a spectral analyser and two voltmeters. The main goal was to construct an integrated system usable for non-expert operators which allows the record of current from fission chambers at high sampling frequency.

With the help of simple experimental set-ups, it has been shown that all the technical requirements needed to record neutron noise were fulfilled. The fission chambers' polarisation voltage is tunable, steady and have a negligible drift during the duration of a typical experiment. Current measurement can be performed with micro-amp and milli-amp calibre. For each calibre of each channel, the mean current measurement is accurate. The electronic transfer function of the system is completely flat on the frequency band of interest, allowing the record and then the computation of the power spectral density without correction or data treatment.

In reactor's experimental results complete the validation of the Spectron system since they are of the order of magnitude of simulation results and are close to results obtained with Feynman- $\alpha$ technique.

In addition to planed measurement on other CEA's experimental reactor, a work is actually underway to assess the sensitivity of computed kinetic parameters to the various procedures of data treatment. It underlines a non negligible variability of the result with data processing parameters and might give some procedure to reduce results' uncertainty.

${ }^{1}$ S. Okajima, T. Sakurai, J. Lebrat, V. Averlant, and M. Martini, Progress in nuclear enegy 41, 285 (2002).

${ }^{2}$ J. C. Carre and J. Da Costa Oliveira, Annals of nuclear Energy 2, 197 (1975)

${ }^{3}$ G. F. Knoll, Radiation Detection and Measurement, 4th Edition ed. (John Wiley \& Sons, 2010).

${ }^{4}$ M. M. R. Williams, Random Processes in Nuclear Reactors, First Edition ed. (Pergamon Press, 1974).

${ }^{5}$ G. I. Bell and S. Glasstone, Nuclear Reactor Theory (Van Nostrand Reinhold Inc.,U.S., 1970)

${ }^{6}$ K. O. Ott and R. J. Neuhold, Introductory Nuclear Reactor Dynamics (American Nuclear Society, 1985).

${ }^{7}$ C. Jammes, B. Geslot, P. Fougeras, R. Rosa, and G. R. Imel, International Journal of Nuclear Energy Science and Technology 2, 213 (2006)

${ }^{8}$ M. S. Bartlett, Nature 161, 686 (1948).

${ }^{9} \mathrm{~J}$. Bendat and A. Piersol, Random Data: Analysis and measurement procedures (Wiley, 2010).

${ }^{10}$ D. Aldridge, Canadian journal of exploration geophysics 28, 62 (1992).

${ }^{11}$ J. Klauder, A. Price, S. Darlington, and W. Albersheim, The bell system technical journal 39, 745 (1960).

${ }^{12}$ G. Bignan, P. Fougeras, P. Blaise, J.-P. Hudelot, and F. Mellier, in Handbook of Nuclear Engineering, edited by D. Cacuci (Springer US, 2010) pp. 2053-2184. 